\title{
Análise morfométrica do acesso temporal lateral para amígdalo-hipocampectomia baseada em imagens de ressonância e tomografia
}

\author{
Tais Siqueira Olmo', Juan Antonio Castro Flores², \\ Carlos Eduardo Roelke ${ }^{3}$, Homero José de Farias e Melo ${ }^{4}$ \\ Hospital São Camilo Santana, São Paulo, SP, Brasil.
}

\section{RESUMO}

Objetivo: Análise morfométrica do acesso lateral para amigdalo-hipocampectomia, com ênfase na localização do "ponto hipocampal". Métodos: Análise de 22 exames de ressonância magnética (RM) e tomografia computadorizada (TC) com o sistema AIMNAV (Micromar Inst), para determinação do ponto hipocampal, e o Advantage Workstation AW 4.3 (GE Medical Systems), para mensuração do corredor cirúrgico. Resultados: O "ponto hipocampal" se localiza a 31,9 mm do canal auditivo. Conclusão: Os dados morfométricos obtidos neste estudo têm utilidade prática na tática da abordagem lateral para amígdalo-hipocampectomia.

\section{PALAVRAS-CHAVE}

Hipocampo/cirurgia, epilepsia do lobo temporal, craniotomia, imagem por ressonância magnética, tomografia.

\begin{abstract}
Lateral approach for amigdalo-hippocampectomy: morphometric data based on MRI and CT scans

Objective: Morphometric analysis of lateral access to amygdalo-hippocampectomy, with emphasis on the location of "hippocampal point". Methods: Analysis of 22 magnetic resonance imaging (MRI) and computed tomography (CT) system with AIMNAV (Micromar Inst) to determine hippocampal point, and the Advantage Workstation AW 4.3 (GE Medical Systems) for measurement of the surgical corridor. Results: The "hippocampal point" is located at $31,9 \mathrm{~mm}$ from the ear canal. Conclusion: The morphometric data obtained in this study have practical utility of the tactical approach to lateral amygdalo-hippocampectomy.
\end{abstract}

\section{KEYWORDS}

Hippocampus/surgery, epilepsy temporal lobe, craniotomy, magnetic resonance imaging, tomography.

Pós-graduanda em Ressonância Magnética da Faculdade Redentor/Instituto Cimas de Ensino, São Paulo, SP, Brasil.

2 Neurocirurgião Hospital São Camilo Santana, Hospital das Clínicas da Faculdade de Medicina da Universidade de São Paulo (HCFMUSP), Irmandade Santa Casa de Misericórdia de São Paulo (ISCMSP), Instituto de Assistência Médica ao Servidor Público Estadual (IAMSPE), São Paulo, SP, Brasil.

3 Neurocirurgião do Hospital São Camilo Santana, IAMSPE, São Paulo, SP, Brasil.

4 Coordenador do Curso de Pós-graduação em Ressonância Magnética da Faculdade Redentor/Instituto Cimas de Ensino, São Paulo, SP, Brasil. 


\section{Introdução}

A epilepsia temporal constitui a forma clínica mais frequente de epilepsia refratária a tratamento medicamentoso. O substrato anatomopatológico é a atrofia do corpo amigdaloide e hipocampo (esclerose mesial temporal EMT). O tratamento cirúrgico constitui a primeira linha de tratamento e objetiva a remoção dessas estruturas (amígdalo-hipocampectomia). ${ }^{1,2}$

Existem várias vias de abordagem (temporal lateral, subtemporal, trans-silviana, cisternal). Em todas é necessária a corticectomia para acesso ao corno temporal do ventrículo lateral.

O mais utilizado é o acesso temporal lateral. A principal complicação dessa via é a quadrantopsia homônima superior por lesão da radiação óptica na parede lateral do ventrículo. ${ }^{3,4}$

Essa complicação pode ser evitada ou minimizada quando a abertura da parede lateral do ventrículo é realizada em sua porção mais anterior e inferior. ${ }^{5-7}$

$\mathrm{Na}$ tática cirúrgica, é importante localizar a projeção topográfica do hipocampo na superfície cutânea e óssea, para planejamento adequado da craniotomia e corticectomia, evitando ou minimizando a lesão da radiação óptica ("acesso seguro").

Nessa etapa, o auxílio dos recursos de imagem é importante. Podem-se realizar mensurações, dissecações, reconstruções em três dimensões de cada estrutura cerebral e manipulações eletrônicas com riqueza de detalhes e alta resolução, facilitando o planejamento da via de abordagem, com a vantagem adicional de se utilizarem imagens de ressonância magnética $(\mathrm{RM}) \mathrm{e}$ tomografia computadorizada (TC) do próprio paciente, representando a situação in vivo e individualizada. ${ }^{8}$

Este estudo objetiva realizar análise morfométrica do acesso lateral para a amígdalo-hipocampectomia, baseada em imagens de RM e TC, para localizar a projeção topográfica da cabeça do hipocampo na superfície cutânea e óssea da região temporal ("ponto hipocampal"), e realizar a mensuração do corredor cirúrgico.

\section{Materiais e métodos}

O trabalho foi aprovado pela Comissão de Ética Médica do Hospital São Camilo Santana em 23 de março de 2012.

Foram selecionados do banco de imagens do nosso Serviço exames RM e TC de encéfalo, de 22 pacientes sem patologia neurológica. $\mathrm{O}$ grupo inclui 15 mulheres e sete homens. A média de idade foi de 30 anos (mínimo 11-máximo 88 anos).

Foi seguido protocolo de adquisições de ressonância no plano axial [TR 12,5, TE 5,3, TI 300, Flip Angle 20, FOV 21X21, THK 0,8 mm, Matriz 256 x 256, 252 imagens e tempo 5:56 min. GE 1.5 T (SIGNA HDX) Release 16.0 bobina: dedicada crânio HNS de oito canais].

As imagens foram convertidas ao formato $3 \mathrm{D}$ e armazenadas no sistema AIMNAV (Micromar Inst.), permitindo a visualização volumétrica, com fusão de imagens de TC e marcação de pontos e regiões de interesse com precisão.

Utilizou-se o programa Target (Micromar Inst.) para obter a projeção topográfica da cabeça do hipocampo na superfície cutânea e na escama do osso temporal. A seguir, foi mensurada a distância desse ponto com o canal auditivo externo, determinando a localização do "ponto hipocampal" (Figura $1 \mathrm{~A}-\mathrm{B}$ ).

Posteriormente, mensurou-se o corredor cirúrgico utilizando o Advantage Workstation AW 4.3 (GE Medical Systems), nas sequências M3D/BRAVO (Brain Volume Imaging), técnica escolhida por proporcionar volume isotrópico do cérebro, com alta resolução; utiliza a técnica ASSET, que diminui o tempo de varredura.

Foi realizado corte coronal na cabeça do hipocampo e desenhado o corredor cirúrgico em formato de triângulo, delimitado pelos seguintes pontos anatômicos:

- A: ponto lateral basal do lobo temporal (representado pela curvatura lateral inferior do lobo temporal);

- B: ponto medial superior (sulco ponto-mesencefálico);
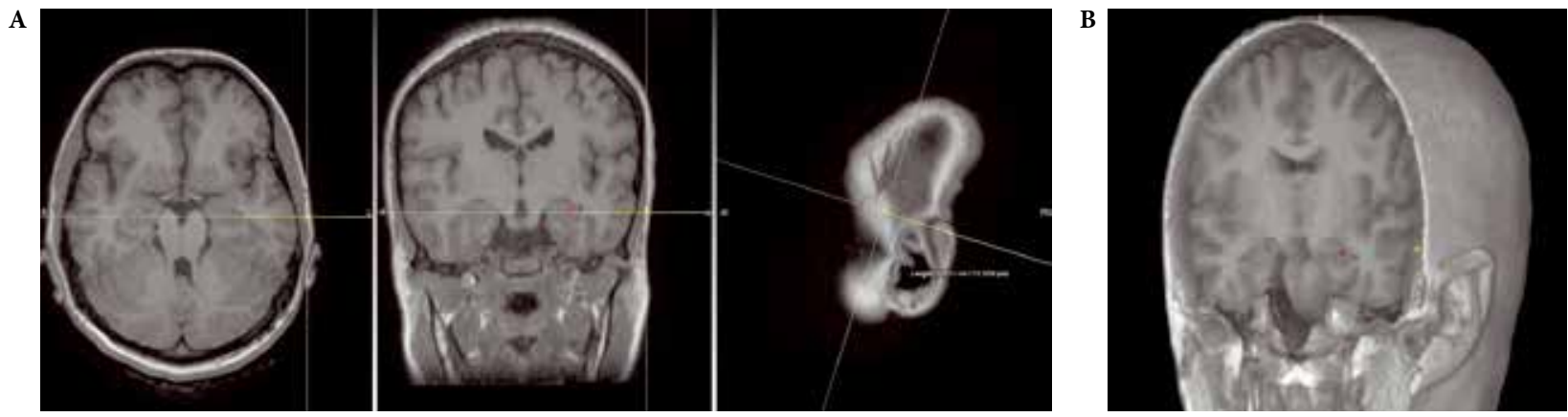

Figura 1 (A-B) - Imagens de RM ilustrando os cortes utilizados no plano axial e coronal para localizar o "ponto". 
- C: ponto medial inferior (borda superior do tronco);

- D: ponto basal (ponto inferior da convexidade temporal basal) (Figura 2).

Foram mensuradas as distâncias entre estes pontos: 1, 2, 3 (Figura 2).

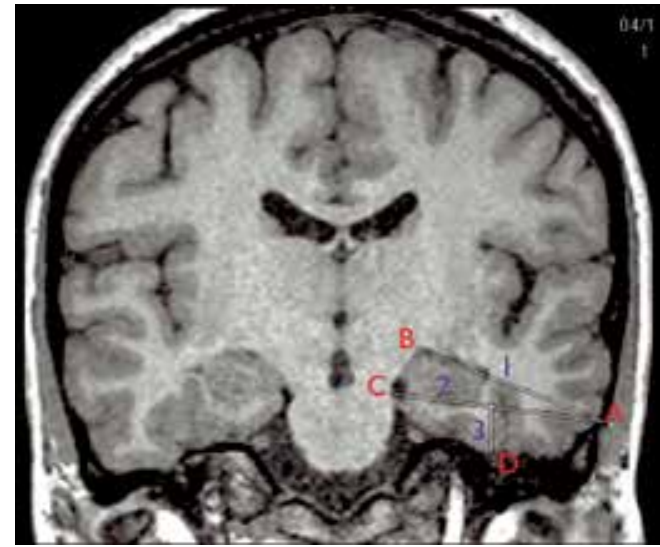

Figura 2 - Corte coronal na topografia da cabeça do hipocampo, ilustrando a mensuração do corredor cirúrgico. (A): ponto lateral basal; (B): ponto medial superior; (C): ponto medial inferior; D: ponto basal.

\section{Resultados}

A distância média (a) canal auditivo-ponto hipocampal foi de 31,9 mm [mínimo (c) 24,7 - máximo (b) $39,6 \mathrm{~mm}$ ] (Figura $3 \mathrm{~A}-\mathrm{B}$ ).

A distância média ponto lateral basal-ponto medial superior 1 foi de 40,9 mm (mínimo 36,3 - máximo 45,1 $\mathrm{mm}$ ) (Figura 2).

A distância média ponto lateral basal-ponto medial inferior 2 foi de 45,8 mm (mínimo 36,8 - máximo 53,4 $\mathrm{mm}$ ) (Figura 2).

A distância média do ponto basal 3 foi de $7,4 \mathrm{~mm}$ (mínimo 4,1 - máximo 10,1 mm) (Figura 2).

Essa última medida representa a profundidade da convexidade temporal basal. Com base nessa medida, foram identificados dois formatos: tipo I - 4,0-8,0 mm (13 pacientes: $59 \%$ ) e tipo II $-8,1-10,1 \mathrm{~mm}$ (9 pacientes: 40\%) (Figuras 4 e 5 ).

\section{Discussão}

Por causa da localização mesial do corpo amigdaloide e hipocampo, as vias de acesso incluem a corticectomia. Algumas complicações resultam dessa primeira etapa. No acesso lateral, a lesão da radiação óptica é frequente.
A

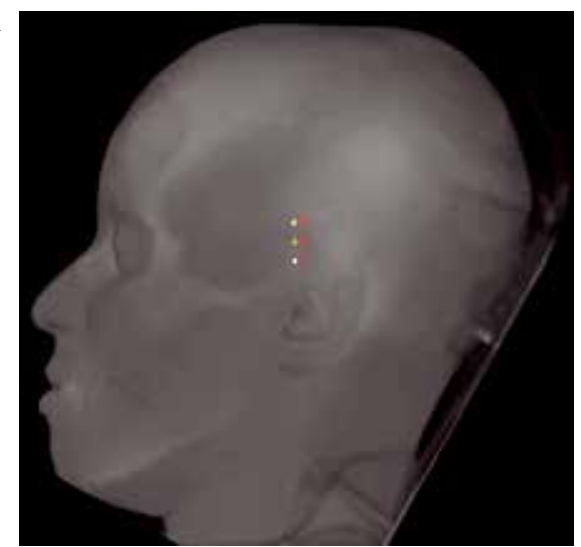

B

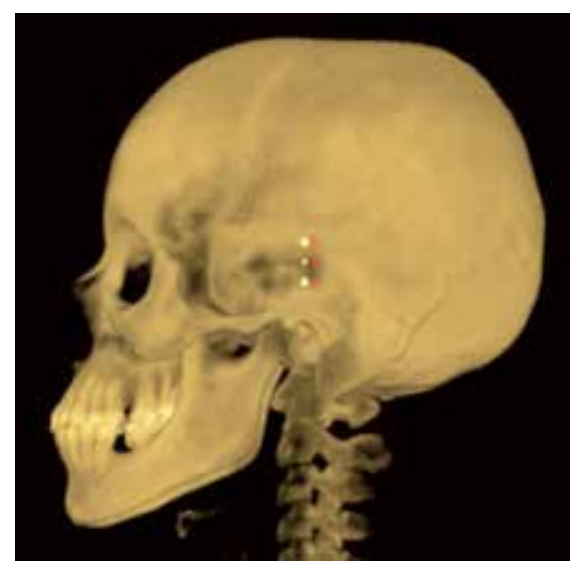

Figura 3 - (A) Localização do "ponto hipocampal” na superfície cutânea e (B) na superfície óssea.

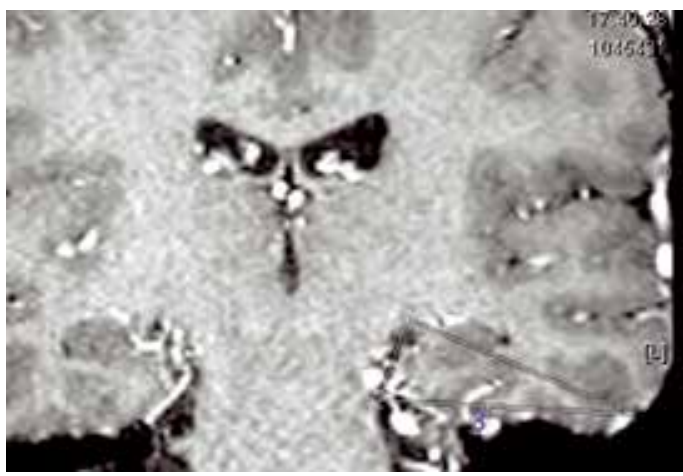

Figura 4 - Convexidade temporal basal tipo 1 (59\%).

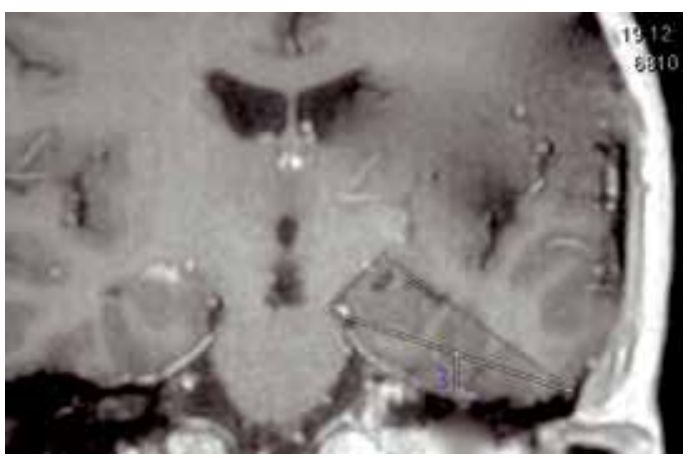

Figura 5 - Convexidade temporal basal tipo II (40\%). 
É possível evitar ou minimizar a lesão da radiação óptica abordando a parede lateral do ventrículo em sua porção mais anterior e inferior ("área livre" de fibras da radiação óptica).

O ponto crucial no planejamento do corredor cirúrgico é a localização da área de projeção da cabeça do hipocampo na superfície cutânea e óssea da região temporal.

Neste estudo, identificou-se esse ponto craniométrico (ponto hipocampal), localizado em média a 31,9 $\mathrm{mm}$ do canal auditivo externo (Figura 3A e B).

O corredor cirúrgico foi delimitado com três pontos anatômicos (Figura 2).

O vértice lateral desse triângulo representa o local da corticectomia. Na base estão as estruturas-alvo (corpo amigdaloide e hipocampo). Esse planejamento permite orientar o acesso ao ventrículo através da porção anteroinferior da parede lateral ("área livre"), evitando a lesão da radiação óptica (Figura 2).

A profundidade da convexidade temporal basal é variável. Foram identificados dois formatos (tipo I e II) (Figuras 4 e 5). É relevante considerar essa classificação no posicionamento cirúrgico da cabeça. No tipo II é recomendada maior deflexão lateral para obter melhor ângulo de acesso (Figuras 4 e 5).

\section{Conclusão}

Os dados morfométricos obtidos neste estudo têm utilidade prática na tática da abordagem lateral para amígdalo-hipocampectomia.

\section{Agradecimentos}

Agradecemos, por sua importante colaboração, ao Sr. Fernando R. Sant’Ana, especialista de Aplicação,
Navegação e Planejamento Cirúrgico Micromar Ind. Com. Ltda.

\section{Referências}

1. Wiebe S, Blume WT, Girvin JP, Eliasziw M. Effectiveness and Efficiency of Surgery for Temporal Lobe Epilepsy Study Group. A randomized, controlled trial of surgery for temporal-lobe epilepsy. N Engl J Med. 2001;345(5):311-8.

2. Schramm J, Clusmann H. The surgery of epilepsy. Neurosurgery. 2008;62(Suppl 2):463-81.

3. Pujari VB, Jimbo H, Dange N, Shah A, Singh S, Goel A. Fiber dissection of the visual pathways: analysis of the relationship of optic radiations to lateral ventricle: a cadaveric study. Neurol India. 2008;56(2):133-7.

4. Egan RA, Shults WT, So N, Burchiel K, Kellogg JX, Salinsky M. Visual field deficits in conventional anterior temporal lobectomy versus amygdalohippocampectomy. Neurology. 2000;55(12):1818-22.

5. Ebeling U, Reulen HJ. Neurosurgical topography of the optic radiation in the temporal lobe. Acta Neurochir (Wien). 1988;92(1-4):29-36.

6. Peuskens D, Van Loon J, Van Calenbergh F, Van den Bergh R, Goffin J, Plets C. Anatomy of the anterior temporal lobe and the frontotemporal region demonstrated by fiber dissection. Neurosurgery. 2004;55(5):1174-84.

7. Silva RC, Flores JAC, Barros MD, Veiga JCE. Estudo anatômico das fibras da radiação óptica no lobo temporal: base para definição de acesso seguro na amigdalohipocampectomia transtemporal. JBNC. 2011;22(1):90.

8. Ardeshiri A, Ardeshiri A, Wenger E, Holtmannspötter M, Winkler PA. Subtemporal approach to the tentorial incisura: normative morphometric data based on magnetic resonance imaging scans. Neurosurgery. 2006;58(Suppl1):ONS22-8.

Endereço para correspondência

Juan Antonio Castro Flores

Rua Prof. Carolina Ribeiro 30, ap. 91, Chácara Klabin

04116-020 - São Paulo, SP, Brasil

Telefone: (11) 2157-7423

E-mail: castroja@me.com 